\title{
Hanseníase no Estado do Pará: expressão do perfil epidemiológico no período de 2006 a 2015
}

Leprosy in the State of Pará: expression of the epidemiological profile in the period from 2006 to 2015

Lepra en el Estado de Pará: expresión del perfil epidemiológico en el período de 2006 a 2015

Waltair Maria Martins Pereira ${ }^{1}$, José Natanael Gama dos Santos ${ }^{1}$, Emanuele Rocha da Silva ${ }^{1}$, Belmiro Figueiredo Vinente Neto ${ }^{1}$, Joao Victor Filgueiras Mota ${ }^{1}$, Yuri Fadi Geha ${ }^{1 *}$, Igor da Silva Narvaes ${ }^{2}$, Marcos Adami², Lourdes Maria Garcez ${ }^{3}$.

\section{RESUMO}

Objetivo: Caracterizar o padrão epidemiológico e identificar as tendências temporais dos indicadores de acompanhamento da hanseníase, estado do Pará, Brasil, de 2006 a 2015. Métodos: Estudo descritivo, de base populacional, com análise de dados do Sistema de Informação de Agravos de Notificação do Ministério da Saúde. Investigaram-se dados clínico-epidemiológicos de 38.859 casos novos. Resultados: Os resultados demonstraram os casos, em geral, do gênero masculino $(60,21 \%)$, de 15 a 59 anos de idade $(75,31 \%)$ e de baixa escolaridade $(66,32 \%)$. A maioria foi conhecida através da demanda espontânea $(56,13 \%)$ e majoritariamente hanseníase multibacilar $(60,94 \%)$, com predomínio da forma clínica dimorfa $(41,56 \%)$, que apresentou tendência de crescimento em projeção até 2020. A magnitude, a força da morbidade e a tendência da doença, permaneceram elevadas, com alta proporcionalidade de casos que apresentam incapacidade física grau II, levantando a suposição de que é elevada a prevalência oculta da doença. A espacialização da taxa média de detecção geral identificou um corredor e dois aglomerados de municípios, com características de muito hiperendemicidade, além de ter evidenciado que 2/3 do Estado permaneceu em hiperendemicidade. Conclusão: Conclui-se que os achados indicaram que a hanseníase é uma endemia persistente no estado do Pará como um sério problema de saúde pública.

Palavras-chave: Hanseníase, Estratégia saúde da família, Doença negligenciada, Epidemiologia descritiva.

\section{ABSTRACT}

Objective: To characterize the epidemiological pattern and identify the temporal trends of indicators for monitoring leprosy, State of Pará, Brazil, from 2006 to 2015. Methods: Descriptive population-based study with data analysis from the Disease Information System Ministry of Health Notification. Clinical and epidemiological data of 38,859 new cases were investigated. Results: The results showed the cases, in general, males $(60.21 \%)$, from 15 to 59 years of age $(75.31 \%)$ and with low education $(66.32 \%)$. Most were known through spontaneous demand $(56.13 \%)$ and mostly multibacillary leprosy $(60.94 \%)$, with a predominance of the borderline clinical form (41.56\%), which showed a growing trend in projection until 2020. The magnitude, the strength of morbidity and the trend of the disease remained high, with a high proportion of cases with grade II physical disability, raising the assumption that the hidden prevalence of the disease is high. The spatialization of the overall average detection rate identified a corridor and two clusters of municipalities, with highly hyperendemic characteristics, in addition to having shown that $2 / 3$ of the state remained in hyperendemicity. Conclusion: It is concluded that the findings indicated that leprosy is a persistent endemic in the State of Pará as a serious public health problem.

Key words: Leprosy, Family health strategy, Neglected disease, Descriptive epidemiology.

1 Universidade Federal do Pará (UFPA), Belém - PA. *E-mail: yuri.geha@ics.ufpa.br

2 Instituto Nacional de Pesquisas Espaciais (INPE), São José dos Campos - SP.

${ }^{3}$ Instituto Evandro Chagas (IEC), Ananindeua - PA. 


\section{RESUMEN}

Objetivo: Caracterizar el patrón epidemiológico e identificar las tendencias temporales de indicadores de seguimiento de la lepra, estado de Pará, Brasil, de 2006 a 2015. Métodos: Estudio descriptivo poblacional con análisis de datos del Sistema de Información de Enfermedades Notificación del Ministerio de Salud. y se investigaron datos epidemiológicos de 38.859 casos nuevos. Resultados: Los resultados mostraron los casos, en general, del sexo masculino $(60,21 \%)$, de 15 a 59 años $(75,31 \%)$ y con bajo nivel educativo $(66,32 \%)$. La mayoría se conocieron por demanda espontánea $(56,13 \%)$ y mayoritariamente por lepra multibacilar $(60,94 \%)$, con predominio de la forma clínica borderline $(41,56 \%)$, que mostró una tendencia creciente en proyección hasta 2020. La magnitud, la fuerza de la morbilidad y la tendencia de la enfermedad se mantuvo alta, con una alta proporción de casos con discapacidad física de grado II, lo que eleva la suposición de que la prevalencia oculta de la enfermedad es alta. La espacialización de la tasa de detección promedio general identificó un corredor y dos conglomerados de municipios, con características altamente hiperendémicas, además de haber demostrado que $2 / 3$ del estado permaneció en hiperendémica. Conclusión: Se concluye que los hallazgos indicaron que la lepra es una endémica persistente en el estado de Pará como un grave problema de salud pública.

Palabras clave: Lepra, Estrategia de salud de la familia, Enfermedad desatendida, Epidemiología descriptiva.

\section{INTRODUÇÃO}

A hanseníase, doença infectocontagiosa crônica causada pelo Mycobacterium leprae ( $M$. leprae), com predileção por células do tecido cutaneomucoso e nervos periféricos, causa lesões que possibilitam o surgimento de incapacidades físicas com grande potencial para serem irreversíveis. É associada à grande carga de estigma, determinando aos doentes dificuldades nas relações sociais, aumentando o processo de estigma e de discriminação, que levam à exclusão social (CRUZ RCS, et al., 2017; PESCADOR MA, et al., 2018).

Para a classificação das formas clínicas da doença, o Ministério da Saúde (MS) (2016a) do Brasil adota as classificações de Madri e a classificação operacional, proposta pela Organização Mundial da Saúde (OMS) (2016a) para o registro da doença no Sistema de Informação de Agravos de Notificação (SINAN). A perspectiva de controle da doença ocorre com a interrupção da transmissão pelo tratamento medicamentoso Poliquimioterápico (PQT), implantado no início da década de 1980.

Segundo a OMS (2016b), a magnitude da hanseníase mantém-se elevada em vários países do mundo. No ano de 2015, nas seis regiões mundiais de notificação e monitoramento da doença, 136 países foram responsáveis pela notificação de 210.750 casos novos, sendo que 11 desses países registraram notificações acima de 1.000 casos. O Continente Americano, com aproximadamente 13\% de todos desses casos notificados, tem o Brasil como o responsável por $92 \%$ dessas notificações.

Embora o Brasil registre decréscimos nas taxas de detecção de casos de hanseníase, em 2015 foram registrados 26.395 casos, com a concentração das áreas mais endêmicas e responsáveis pela manutenção da transmissão localizadas nas regiões Norte, Nordeste e Centro-Oeste do país (OMS, 2016b; MS, 2016b).

A OMS definiu a meta de eliminação da hanseníase como problema de saúde pública, quando a prevalência fosse registrada em menos de um caso por 10 mil habitantes, além de periodicamente lançar novas diretrizes para estimular os países de maior notificação de casos a enfrentar estratégias para o controle da doença. O MS (2016a), em consequência da complexidade e da manutenção da transmissão da doença, vem aderindo aos novos compromissos sem alcançá-los integralmente e enfrenta grandes desafios para o controle da doença.

Para o período de 2016 a 2020, está em curso a Estratégia Global contra a Hanseníase, cujos princípios fundamentais incluem a detecção precoce de todos os pacientes, antes de instalada a deficiência física; a redução da taxa de casos de Grau II de Incapacidade Física (GIF II) para menos de 1/ 1.000.000 hab.; a realização do tratamento imediato com PQT; o favorecimento da inclusão social de pessoas afetadas; o 
reforço da investigação de casos suspeitos e a ampliação de parcerias com outras instituições, transformando as atividades de controle da doença em ações interinstitucionais, e mais uma vez o Brasil aderiu a esse importante compromisso na busca do controle da doença (OMS, 2016a).

No estado do Pará, no ano de 2015 , de acordo com os parâmetros do MS, a avaliação da força, da magnitude da doença e da tendência da endemia, realizadas pela análise da taxa de detecção geral $(35,34$ por 100 mil habitantes) classificou o Estado como área de muito alta endemicidade. Entretanto, na avaliação da força da transmissão recente da endemia a classificação foi de área de hiperendemicidade, visibilizada pela taxa de deteç̧ão em menores de 15 anos de idade de 13,32 por 100 mil habitantes (MS, 2016b).

Devido à baixa disponibilidade de estudos sobre o comportamento e a distribuição da hanseníase em um Estado brasileiro com forte tendência histórica a hiperendemicidade, bem como com a necessidade de ofertar subsídios para o desenvolvimento e a intensificação das medidas de controle, este estudo objetivou caracterizar o padrão epidemiológico e identificar as tendências temporais dos indicadores de acompanhamento da hanseníase em um estado da região Amazônica brasileira (Pará), em uma década de análise.

\section{MÉTODOS}

Trata-se de uma pesquisa quantitativa com desenho de estudo descritivo de base populacional. $O$ estudo se desenvolveu no estado do Pará, situado na Amazônia brasileira, que por ocasião do Censo 2010, contava com uma população de 7.581 .051 habitantes (50,41\% do gênero masculino e $49,59 \%$ do feminino), com $68,48 \%$ residentes em áreas urbanas. No ano de 2015 , apresentou aumento da população para 8.104 .880 habitantes, sendo 50,5\% (4.092.964) do gênero masculino e 49,5\% (4.011.916) do gênero feminino. Apresenta extensão territorial de $1.247 .689,76 \mathrm{~km}^{2}$, ocupa $15 \%$ do território nacional e $45 \%$ do território da Região Norte (COSTA EJM, 2011; INSTITUTO BRASILEIRO DE GEOGRAFIA E ESTATíSTICA (IBGE), 2010).

Os dados demográficos e epidemiológicos dos casos foram obtidos das fichas de notificação e investigação de hanseníase, registradas no Sistema de Informação de Agravos de Notificação (SINAN) e arquivadas na Secretaria de Estado de Saúde Pública do Pará (SESPA) (2017), sendo incluídos os registros de todos os casos notificados como de hanseníase e residentes no estado do Pará e excluídos aqueles cujo diagnóstico da doença não foi confirmado. Considerando que o município de Mojuí dos Campos, até o ano de 2012, pertenceu a jurisdição do município de Santarém, os dados desse município, para o primeiro período bianual da distribuição geográfica da taxa de detecção foram incluídos no município de Santarém.

Os dados relativos às variáveis epidemiológicas e demográficas, foram aqueles utilizados para a construção dos indicadores epidemiológicos que medem a força da morbidade, a magnitude e a tendência da doença, recomendados pelo MS e disponibilizados nas Diretrizes para vigilância, na atenção e eliminação da hanseníase como problema de saúde pública (MS, 2016a).

Para a variável raça/cor da pele foram seguidas as especificações do Estatuto da Igualdade Racial (Lei $n^{\circ}$ 12.288/2010), que define a população negra como o "conjunto de pessoas que se autodeclararam pretas e pardas, conforme o quesito cor raça, usado pelo IBGE (2014)". Tendo em vista que as categorias de raça/cor da pele utilizadas pelo SINAN serem as mesmas classificadas pelo IBGE, nesta pesquisa, os casos considerados como negros foram aqueles indivíduos que se autodeclararam pretos, somados aqueles que se autodeclararam pardos.

Os parâmetros utilizados para a classificação da endemicidade foi seguido o que é preconizado nas Diretrizes para vigilância, atenção e eliminação da hanseníase como problema de saúde pública, recomendadas pelo MS (2016a). Porém, devido aos elevados valores das taxas de detecção de hanseníase em muitos municípios do Estado, foi adicionada uma nova classificação, denominada "muito hiperendêmico", que engloba o valor da taxa de detecção geral superior a 100 casos por 100.000 habitantes, seguindo o mesmo critério adotado na pesquisa que estudou a correlação espacial da hanseníase com a migração, nos municípios do estado do Pará (PEREIRA WMM, et al., 2021). 
Os dados demográficos e cartográficos de identificação dos limites municipais foram aqueles disponibilizados em fontes secundárias oficiais pertencentes às diversas esferas de governo nos níveis Federal, Estadual e Municipal, de domínio público, que compõem a base de dados do IBGE, dos censos referentes aos anos de 2000 e 2010 e as estimativas populacionais para os anos intercensitários, 2006 a 2009 e 2011 a 2015 (IBGE, 2000; IBGE, 2010). As análises da distribuição geográfica das taxas de detecção de hanseníase na população geral foram aglutinadas em 5 períodos bianuais (2006 a 2007, 2008 a 2009, 2010 a 2011, 2012 a 2013 e 2014 a 2015) para o controle da influência de números extremos sobre as taxas, para mais ou para menos, bem como para o período total.

$\mathrm{Na}$ estatística descritiva, para a análise da distribuição de frequências, foi aplicado o teste qui-quadrado, para melhor observar o ajuste, a homogeneidade e a independência da variável, através do software BioEstat, versão 5.0 (AYRES M, et al., 2007). Para o cálculo da tendência linear das taxas de detecção geral e em menores de 15 anos; taxa de prevalência; proporção da hanseníase por classificação clínica; proporção de casos multibacilares; proporção de GIF II no momento do diagnóstico e cobertura populacional da ESF foi

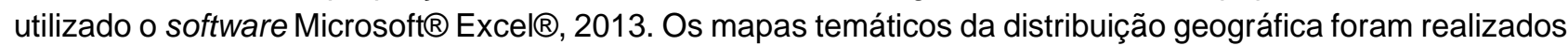
no software livre com código-fonte aberto (QGis) 2.18, que permitiu a interação com os bancos de dados e a aplicação em Sistemas de Informações Geográficas (GIS).

O estudo foi aprovado pelo Comitê de Ética e Pesquisa (CEP) do Instituto de Ciências da Saúde (ICS) da UFPA, com Certificado de Apresentação para Apreciação (CAAE) n 33158219.5.0000.0018; Parecer $n^{\circ}$ 4.146.997, de 9 de julho de 2020.

\section{RESULTADOS}

Foram notificados 38.859 casos novos de hanseníase no período de 2006 a 2015, em todo o estado do Pará. A maioria residia na área urbana dos municípios (69,92\% - 27.169/38.859), com idade entre 15 e 39 anos $(46,74 \%$ - 18.161/38.859) e 75,31\% (29.264/38.859) pertenciam à faixa etária economicamente ativa (15 a 59 anos). Uma parcela significativa dos casos declarou ter cor da pele preta e/ou parda $(80,45 \%$; $31.261 / 38.859)$ e eram do gênero masculino (60,21\%; 23.396/38.859), na comparação entre as categorias das variáveis estudadas $(p<0,0001)$. Foi alta a frequência de indivíduos com baixo nível de escolaridade $(66,32 \% ; 25.771 / 38.859)(p<0,0001)$.

Muitos casos foram conhecidos através da demanda espontânea $(56,13 \% ; 21.813 / 38.859)(p<0,0001)$. Os exames da coletividade e de contatos corresponderam a 9,46\% (3.675/38.859) do total de casos detectados. As taxas padronizadas de detecção geral e em menores de 15 anos e a taxa de prevalência da hanseníase apresentaram um histórico de declínio mais pronunciado para a taxa de detecção geral (Gráfico 1). 
Gráfico 1 - Tendência da detecção geral e em menores de 15 anos e da prevalência da hanseníase no estado do Pará, Brasil, 2006 a 2015.

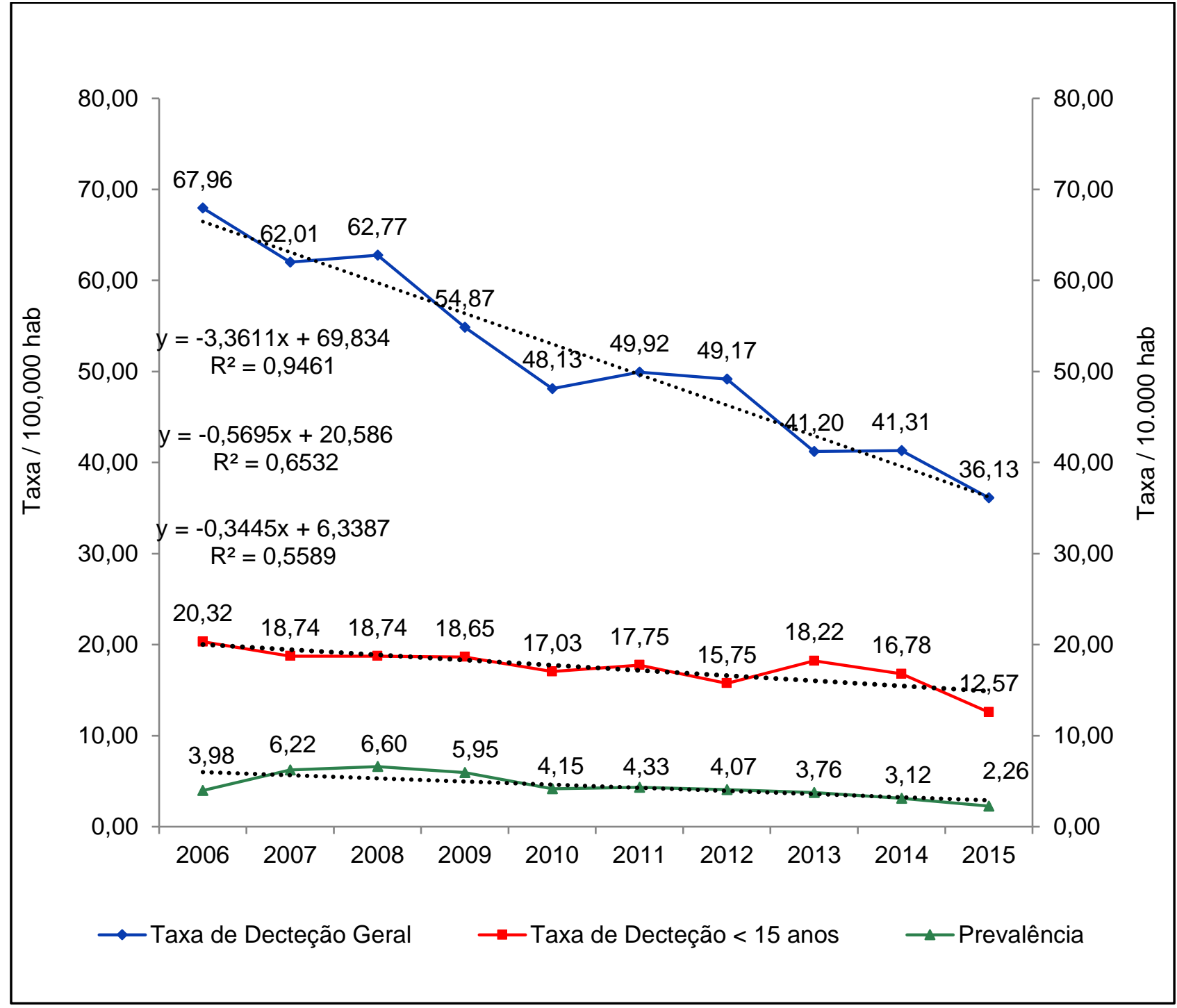

Fonte: Pereira WMM, et al., 2021; dados extraídos do SINAN, 2016.

A classificação operacional da hanseníase apontou crescimento contínuo das formas Multibacilares (MB) quando comparadas, ano a ano, com os casos das formas Paucibacilares (PB), as quais demonstram padrão decrescente (Gráfico 2). 
Gráfico 2 - Hanseníase, segundo a classificação operacional, no estado do Pará, Brasil, 2006 a 2015.

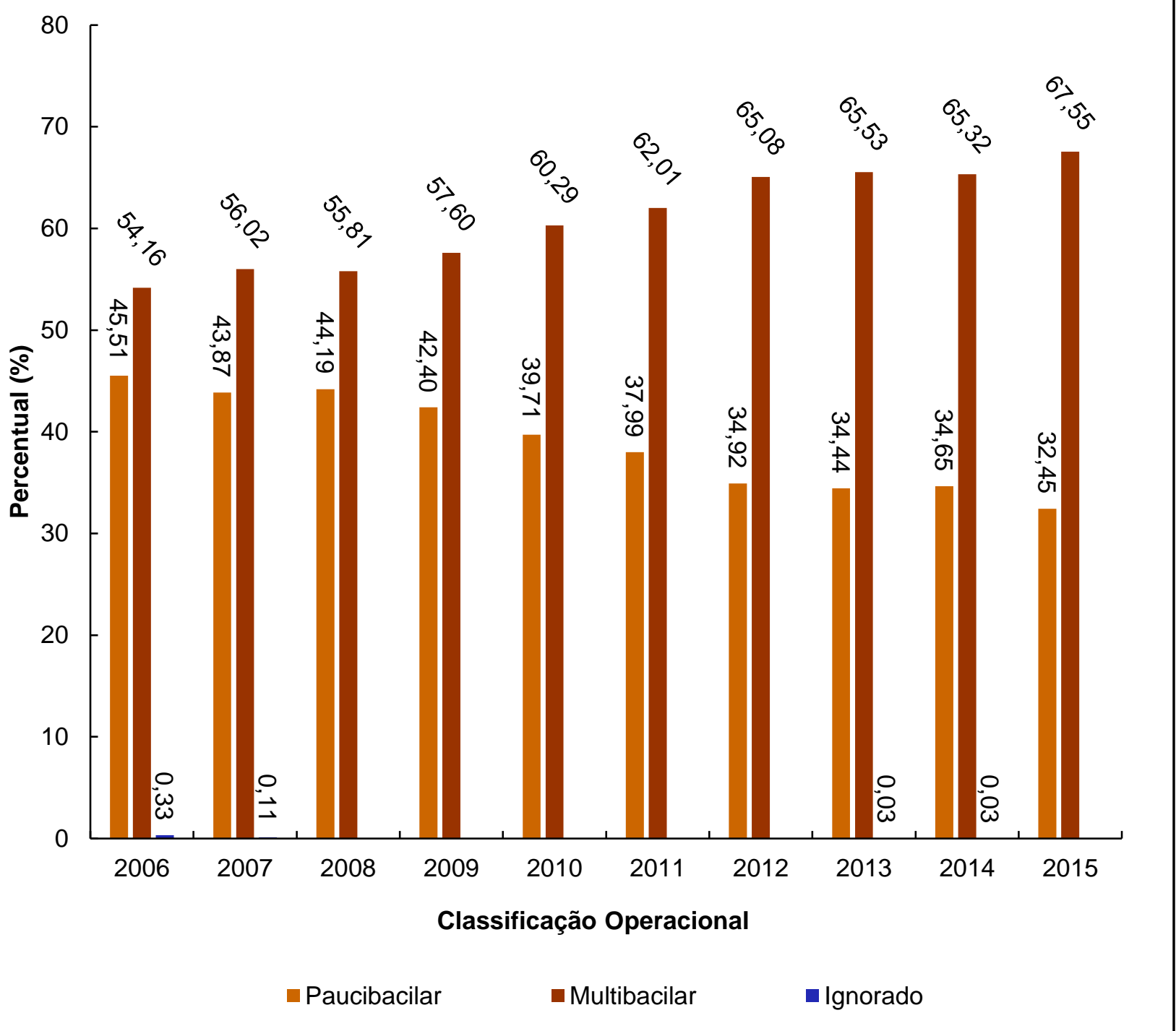

Fonte: Pereira WMM, et al., 2021; dados extraídos de SINAN, 2016.

A análise da tendência da proporcionalidade das formas clínicas de hanseníase tornou claro que a forma dimorfa foi a de maior proporcionalidade, seguida pela forma clínica indeterminada. A predição da tendência linear para os cinco anos seguintes ao período de estudo (2016 a 2020), se observa que a forma dimorfa se manterá em maior proporcionalidade, inclusive sendo a única forma clínica que registrará crescimento, com a maior aderência entre os registros em relação aos valores estimados pela equação $\left(R^{2}=0,8119\right)($ Gráfico 3). 
Gráfico 3 - Tendência linear, da proporção da hanseníase, por classificação clínica, para o período de 2006 a 2015 e predição da tendência, por classificação clínica no período de 2016 a 2020.

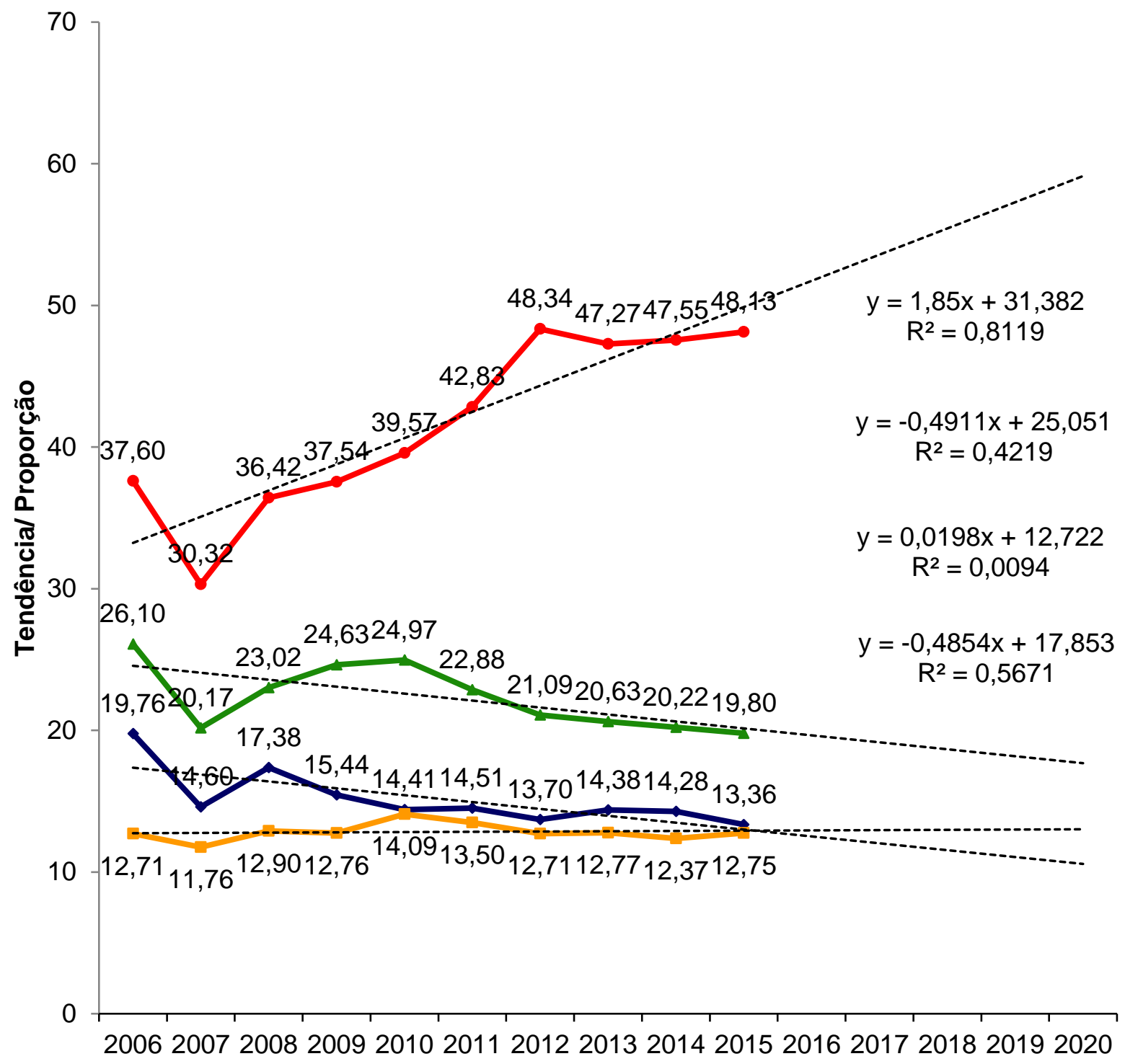

Formas clínicas

-Indeterminada $\rightarrow$ Tuberculóide $\rightarrow$ Dimorfa $\quad$ Virchowiana

Fonte: Pereira WMM, et al., 2021; dados extraídos de SINAN, 2016.

Em que pese à taxa de detecção geral da hanseníase vir apresentando tendência decrescente ano após ano, a proporcionalidade de casos multibacilares, apresentou tendência de expressivo crescimento, entretanto os casos multibacilares que apresentaram grau de incapacidade física tipo II apresentaram tendência de estabilização em valores considerados baixos ao longo de todo o período de análise (Gráfico 4). 
Gráfico 4 - Tendência da detecção geral da hanseníase e tendência da proporção de casos novos de hanseníase multibacilares e de casos multibacilares com GIF II, no momento do diagnóstico. Estado do Pará, Brasil, 2006 a 2015.

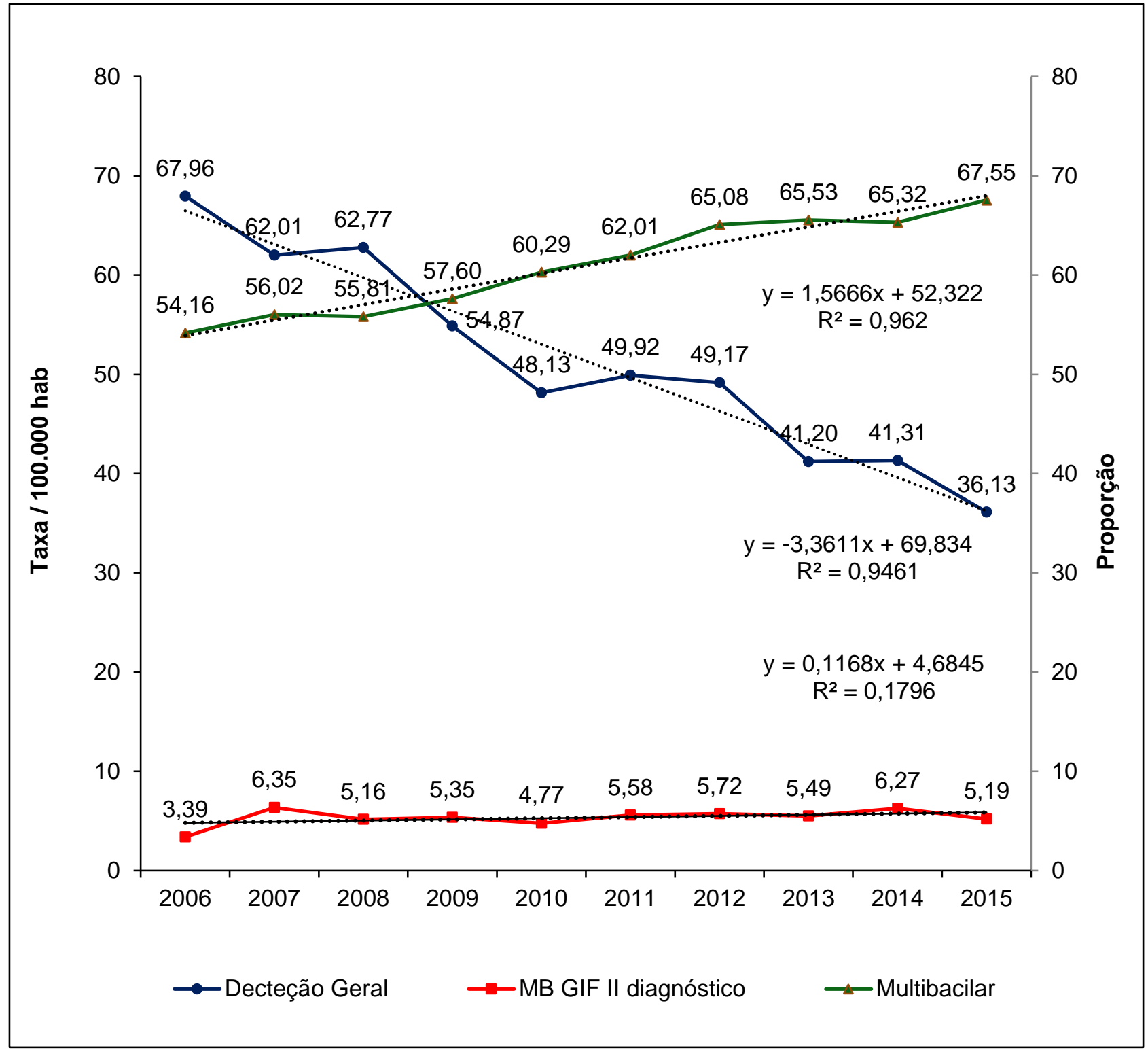

Fonte: Pereira WMM, et al., 2021; dados extraídos de SINAN, 2016

A espacialização das taxas médias, padronizadas, de detecção de hanseníase, aponta heterogeneidade a cada biênio estudado, demonstrando regressão das áreas classificadas como "muita hiperendêmicas" para áreas com hiperendemicidade. A análise das taxas aglutinadas para todo o período de estudo (2006-2015), identificou uma concentração contígua, caracterizando um corredor de transmissão composto por 14 municípios, que percorre as Mesorregiões Sudoeste e Sudeste e ainda conforma dois aglomerados, sendo um com 6 municípios e o outro com 3 municípios, com taxas classificadas como "muito hiperendêmicas", localizados integralmente na Mesorregião Sudeste. A hiperendemicidade da doença se apresentou de forma tão extensa que atingiu cerca de $2 / 3$ da área territorial do Estado em todos os períodos bianuais e no total geral da série histórica estudada (Figura 1). 
Figura 1 - Distribuição geográfica das taxas médias de detecção geral da hanseníase, períodos bianuais e total estudados. Municípios do estado do Pará, Brasil, período 2006 a 2015.

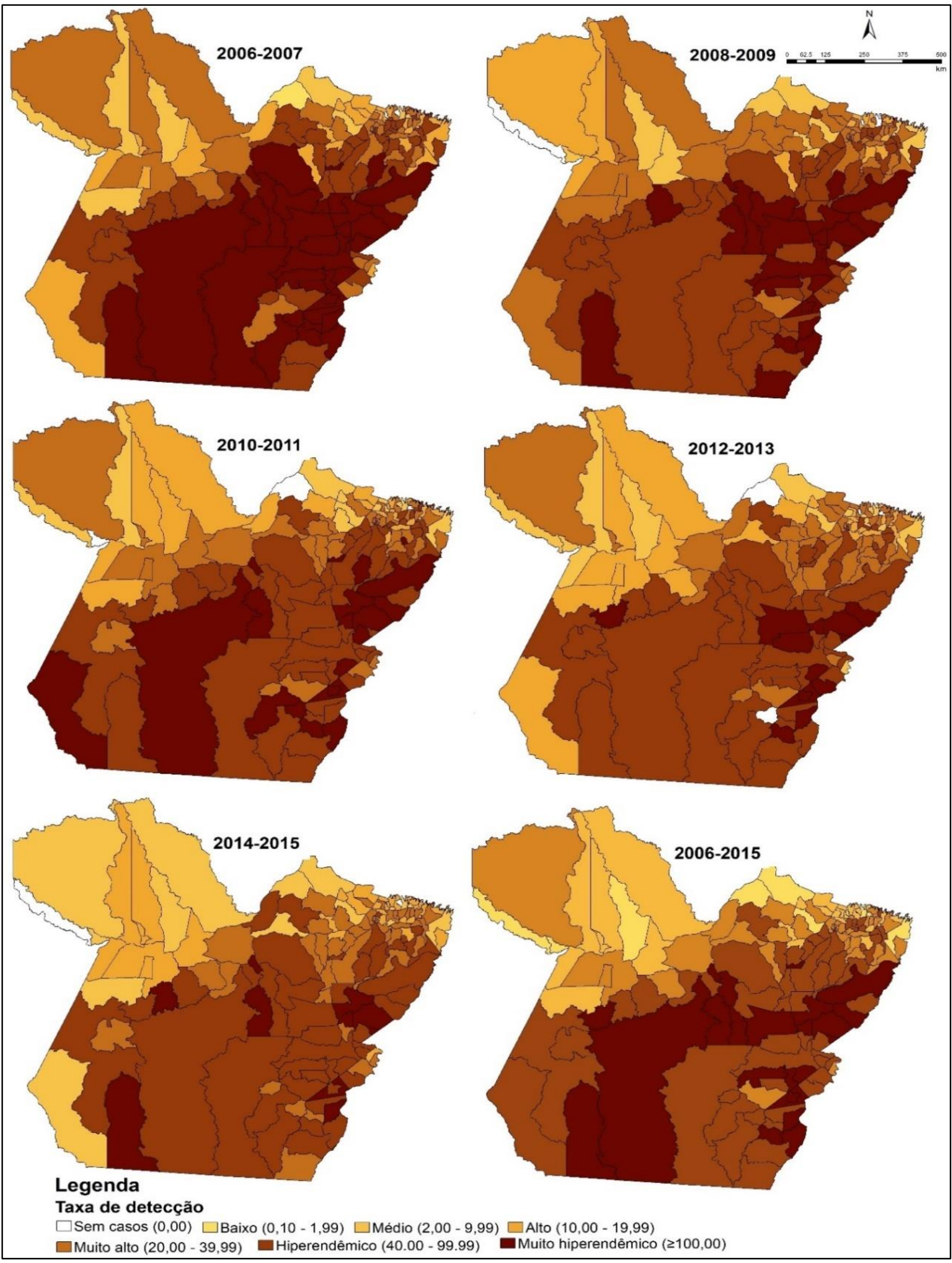

Fonte: Pereira WMM, et al., 2021; dados extraídos de SINAN, 2016; Software QGis 2.18. 


\section{DISCUSSÃO}

Os resultados apresentados neste estudo identificaram uma alta proporção de casos de hanseníase multibacilar, da forma clínica dimorfa, do sexo masculino e em faixa etária economicamente ativa. As taxas de detecção geral e em menores de 15 anos, assim como a taxa de prevalência, apresentaram tendência decrescente para o período de análise. A heterogeneidade espacial foi evidente, identificando as áreas de alto risco para a transmissão da doença, ocupando as Mesorregiões Sudoeste, Sudeste e parte das Mesorregiões Nordeste e do Marajó (PEREIRA WMM, et al., 2021).

A maior ocorrência de hanseníase no sexo masculino com razão de masculinidade de 1,51 casos em homens em relação a 1 caso em mulheres, não difere de pesquisas anteriores realizadas no Brasil, que encontraram variação na detecção entre os gêneros, com maior predomínio para os homens (RIBEIRO LCG, et al., 2021). As maiores frequências da doença, ocorridas nas faixas etárias de 15 a 39 anos e de 40 a 59 anos (46,74\% e $28,57 \%$, respectivamente), também não diferem do que já foi encontrado em Ribeiro LCG, et al. (2021).

O predomínio de portadores de hanseníase que declararam ter a cor da pele preta e/ou parda $(80,45 \%$, pvalor $<0,0001$ ) ficou bem próximo do que foi identificado por ocasião do censo de 2010 , quando as pessoas se autodeclararam negros e/ou pardos (76,5\%). Esse achado evidencia as desigualdades existentes nesse segmento populacional, que, historicamente, representa a maior parte da população do país e que mais sofre com as desigualdades advindas da vida social e econômica da estrutura social a que pertença, incluindo, inclusive, as dificuldades para o acesso ao sistema de saúde (ABRITA APCT e ABRITA MB, 2018; PASSOS ALV e ARAÚJO LF, 2020).

A maior frequência $(66,32 \%$; $p$-valor $<0,0001)$ de casos sem escolaridade e/ou que tinham apenas o ensino fundamental incompleto reforça a hipótese de que, quanto mais baixo o nível de escolaridade, maiores são as dificuldades socioeconômicas, menores são as possibilidades de acesso a bens e serviços e maiores são as chances para adoecer de hanseníase. Desta forma, estudos comprovam a hipótese de que os determinantes sociais exercem papel central no processo de adoecimento, em particular para a hanseníase no estado do Pará (NERY JS, et al., 2019; RIBEIRO GDC e LANA FCF, 2015).

A maior proporcionalidade de novos casos detectados em áreas urbanas $(69,92 \% p$-valor $<0,0001)$ não difere da caracterização demográfica do estado do Pará, identificada no censo de 2010 , onde registrava que $68,48 \%$ da população residia em áreas urbanas (IBGE, 2012). Assim, a expansão da doença nos grandes e médios municípios do Estado, principalmente nas áreas urbanas, deveu-se ao crescimento da periferia das cidades, provavelmente, devido ao fluxo migratório interno, impulsionado pela busca de oportunidades de emprego e por melhores ofertas de ensino para membros da família para aqueles que tradicionalmente residiam nas áreas rurais (SESPA, 2009).

A maior detecção de casos novos por demanda espontânea $(56,13 \%$; p-valor $<0,0001)$ demonstrou que os serviços de saúde estavam realizando a detecção de casos de forma passiva, quer por baixa eficiência dos serviços ou quer ainda devido as baixas coberturas populacionais da Estratégia Saúde da Família no Estado, que dificultam a expressão da busca ativa de casos e o diagnóstico precoce (MONTEIRO LD, et al., 2018; OLVEIRA DJS, et al., 2021).

A baixa detecção de casos $(9,46 \%)$ através de exame da comunidade e dos contatos de casos novos sinaliza a fragilidade das atividades de busca ativa. Pesquisas anteriores, realizadas no estado do Pará, identificaram que a maioria dos casos de hanseníase foi encontrada em famílias numerosas, reforçando a necessidade de que estratégias sejam desenvolvidas para alcançar uma maior cobertura do exame dos contatos, o que ajudaria na queda da transmissão da doença, auxiliaria no diagnóstico precoce e reduziria o número de pessoas portadoras de incapacidades físicas decorrentes do atraso no diagnóstico (RIBEIROFILHA C, et al., 2017; BLOK DJ, et al., 2015; OLIVEIRA KS, et al., 2015).

A frequência crescente de casos novos nas formas multibacilares é um indicativo de que a doença está sendo detectada tardiamente e que a cadeia de transmissão do $M$. leprae é mantida na população. Essa 
situação serve de alerta por serem estas as formas clínicas que potencialmente causam casos com GIF II no momento do diagnóstico (GOIABEIRA YNLA, et al., 2018).

Quando analisados separadamente, os indicadores epidemiológicos apontam graus distintos de endemicidade no Pará. Assim, a taxa de prevalência em declínio classificou o Estado como área que apresentou média endemicidade para hanseníase, divergindo da taxa de detecção geral, que o classificou como área geográfica de "muito endemicidade" (MS, 2016a; OLVEIRA DJS, et al., 2021). A taxa de detecção em menores de 15 anos com índice de hiperendemicidade deixou claro que o Estado apresentava grande força na transmissão recente da doença, desenhando alta endemicidade, similar ao encontrado no estudo de Pires CAA, et al. (2019), no período de 2010 a 2015 no estado do Pará.

A distribuição geográfica das taxas média e geral de hanseníase, padronizadas por idade, para todos os municípios do Estado, permitiu identificar que foi permanente a diminuição do número de municípios classificados na categoria "muito hiperendêmico" ( $\geq 100 / 100.000$ hab), entretanto não afastou o Pará de apresentar mais de $2 / 3$ de sua área territorial, com os municípios classificados como "hiperendêmicos" (40 a $\leq 100 / 100.000 \mathrm{hab}$ ) (PIRES CAA, et al., 2019; PEREIRA WMM, et al., 2021).

A identificação de um corredor de municípios no sentido da Mesorregião Sudoeste para a Sudeste, com taxas de detecção classificadas como de "muito hiperendemicidade", coincidido com a área onde estão implantados projetos de extração mineral e de desenvolvimento da agropecuária, sinalizam para a importância do processo migratório, fazendo parte da associação da multiplicidade de fatores que facilitam a transmissão da doença (PEREIRA WMM, et al., 2021).

A formação de dois aglomerados de municípios "muito hiperendêmicos", localizados na Mesorregião Sudeste do estado do Pará, conformando a área impactada pelo projeto de implantação da Hidroelétrica de Tucuruí e em área fronteiriça com o estado de Tocantins, sabidamente área das mais altas taxas de detecção e de prevalência da doença entre os estados da Região Norte, também sinalizou para a importância do processo migratório como influenciador dos altos índices das taxas de detecção da doença (PEREIRA WMM, et al., 2021).

Diante da utilização de base de dados secundários (SINAN), reconhece-se a presença de limitações inerentes ao estudo realizado, como a qualidade e a fidedignidade do preenchimento das fichas de notificação e investigação, bem como a subnotificação dos casos. Desse modo, reitera-se a sua importância para a complementação dos conhecimentos obtidos e apresentados, em busca por uma melhor integração ensino/serviço, maior execução de pesquisas de base populacional para a ampliação do conhecimento adequado da situação epidemiológica da hanseníase, assim como a sustentação do planejamento da execução oportuna de atividades de vigilância epidemiológica, de expansão da cobertura populacional de ESF e do investimento em capital intelectual sobre o controle da doença.

\section{CONCLUSÃO}

Os resultados encontrados alertam que a detecção de casos está sendo realizado de forma tardia, permitindo que focos da doença permaneçam ativos por muito tempo na comunidade, contribuindo para levantar a hipótese de que a prevalência oculta da doença é alta no estado do Pará. A heterogeneidade espacial foi evidente ao identificar as áreas de alto risco para a transmissão da doença, com taxas de detecção hiperendêmicas que se estendem por mais de 2/3 do território do Estado e com a permanência da taxas "muito hiperendêmicas" nas áreas onde existem projetos de extração mineral e agropecuários, com rodovias estaduais importantes para o trânsito das pessoas que procuram trabalho e renda, e que, ao chegarem aos novos territórios, ocupam-no de forma desordenada e com dificuldades socioeconômicas, o que traz grande aviltamento para suas qualidades de vida. Dessa forma, os achados provenientes desta pesquisa permitiram caracterizar a hanseníase como um relevante problema de saúde pública no estado do Pará, o qual serve como subsídio para demais estudos em outras regiões da Amazônia. 


\section{REFERÊNCIAS}

1. ABRITA APCT, ABRITA MB. Hanseníase: aspectos psicológicos e socioeconômicos. Brazilian Journal of Scientific Administration, 2018; 9(1): 81-92.

2. AYRES M, et al. BioEstat 5.0: aplicações estatísticas nas áreas das ciências biológicas e médicas. Belém: Sociedade Mamirauá, 2007; 364p.

3. BLOK DJ, et al. Global elimination of leprosy by 2020: are we on track? Parasit Vectors, 2015 Oct 22; 8: 548.

4. COSTA EJM. Planejamento Territorial, Gestão de Políticas Públicas e Descentralização Regional: a experiência do Estado do Pará. DRd - Desenvolvimento Regional Em Debat, 2011; 1(1): 120-148.

5. CRUZ RCS, et al. Hanseníase: situação atual, aspectos clínicos, laboratoriais, histórico do tratamento e perspectiva de esquema único para todas as formas clínicas. An Bras Dermatol. 2017; 92(6):764-77.

6. GOIABEIRA YNLDA, et al. Perfil Epidemiológico e clínico da hanseníase em capital hiperendêmica. Rev. enferm. UFPE online, 2018; 12(6): 1507-1513.

7. INSTITUTO BRASILEIRO DE GEOGRAFIA E ESTATÍSTICA (IBGE). Censo demográfico 2000. Disponível em: https://www.ibge.gov.br > 9663-censo-demografico-2000. Acessado em: 08 de abril de 2021.

8. INSTITUTO BRASILEIRO DE GEOGRAFIA E ESTATÍSTICA (IBGE). Censo demográfico 2010. Disponível em: https://censo2010.ibge.gov.br. Acessado em: 08 de abril de 2021.

9. INSTITUTO BRASILEIRO DE GEOGRAFIA E ESTATÍSTICA (IBGE). Estatística/população/resultados gerais/amostras resultados gerais. 2012. Disponível em: https://biblioteca.ibge.gov.br > livros > liv98965. Acessado em: 8 de abril de 2021.

10. INSTITUTO BRASILEIRO DE GEOGRAFIA E ESTATÍSTICA (IBGE). Estatísticas de gênero. Notas técnicas. Fundamento legal e sigilo das informações. 2014. Disponível em: https://www.ibge.gov.br/apps/snig/v1/notas_metodologicas.html?loc=0. Acessado em: 8 de abril de 2021.

11. MINISTÉRIO DA SAÚDE (MS). Secretaria de Vigilância em Saúde. Departamento de Vigilância das Doenças Transmissíveis. Diretrizes para vigilância, atenção e eliminação da Hanseníase como problema de saúde pública: manual técnico-operacional [recurso eletrônico]. Brasília: Ministério da Saúde, Brasil, 2016a. Disponível em: https://portalarquivos2.saude.gov.br. Acessado em: 8 de abril de 2021.

12. MINISTÉRIO DA SAÚDE (MS). Secretaria de Vigilância em Saúde. Indicadores epidemiológicos e operacionais de hanseníase, Brasil, 2000-2015. Brasília: Ministério da Saúde, Brasil, 2016b. Disponível: https://antigo.saude.gov.br. Acessado em: 8 de abril de 2021.

13. MONTEIRO LD, et al. Tendências da hanseníase após implementação de um projeto de intervenção em uma capital da Região Norte do Brasil, 2002-2016. Cad. Saúde Pública. 2018; 34(11): e00007818.

14. NERY JS, et al. Socioeconomic Determinants os Leprosy New Case Detection in the 100 Million Brazilian Cohort: a Population-Based Linkage Study. Lancet Global Health. 2019; 7: e1226-e1236.

15. OLIVEIRA DJS, et al. Perfil espacial e demográfico da Hanseníase no norte do país, no período de 2014 a 2019. Revista Eletrônica Acervo Saúde. 2021; 13(4): e7145.

16. OLIVEIRA KS, et al. Avaliação dos indicadores epidemiológicos e operacionais para a hanseníase em municípios prioritários no estado do Paraná, 2001 a 2010. Epidemiol. Serv. Saúde. 2015; 24(3): 507-516.

17. ORGANIZAÇÃO MUNDIAL DA SAÚDE (OMS). Global Escritório Regional para o Sudeste Asiático. Estratégia global para a hanseníase: acelerar para um mundo livre de hanseníase, período 2016 a 2020. 2016a. Disponível em: https://apps.who.int. Acessado em: 08 de abril de 2021.

18. ORGANIZAÇÃO MUNDIAL DA SAÚDE (OMS). Global leprosy update, 2015: time for action, accountability and inclusion. Weekly Epidemiol Rec. 2016b. Disponível em: https://www.who.int ı. Acessado em: 8 de abril de 2021.

19. PASSOS ALV, ARAÚJO LF. Representações sociais da hanseníase: um estudo psicossocial com moradores de um antigo hospital colônia. INTERAÇÕES. 2020; 21 (1): 93-105.

20. PEREIRA WMM, et al. Hanseníase e migração: Correlação espacial em um Estado hiperendêmico da Amazônia brasileira. Research, Society and Development. 2021; 10(1): 1-12.

21. PESCADOR MA, et al. Análise de tendência histórica da evolução da hanseníase em Santa Catarina no período de 2001-2015. Arq. Catarin Med. 2018; 47(1): 141-158.

22. PIRES CAA, et al. Análise do perfil clínico- epidemiológico da hanseníase no Pará e avaliação dos indicadores de saúde, Revista Eletrônica Acervo Saúde, 2019; 27: e899.

23. RIBEIRO GDC e LANA FCF. Incapacidades físicas em hanseníase: caracterização, fatores relacionados e evolução. Cogitare Enferm. 2015; 20(3): 496-503.

24. RIBEIRO LCG, et al. Características demográficas e clínicas do grau de incapacidade física associadas ao diagnóstico e alta do tratamento da hanseníase. 2021; Revista Eletrônica Acervo Saúde 13(2): e6008.

25. RIBEIRO-FILHA C, et al. Hanseníase. In: Ministério da Saúde (BR), Secretaria de Vigilância em Saúde, CoordenaçãoGeral de Desenvolvimento da Epidemiologia em Serviços. Guia de vigilância em saúde [Internet]. Brasília: Ministério da Saúde, 2017; 309-340. Disponível em: https://bvsms.saude.gov.br/bvs/publicacoes/guia_vigilancia_saude_volume_2.pdf. Acessado em: 8 de abril de 2021.

26. SECRETARIA DE ESTADO DE SAÚDE PÚBLICA DO PARÁ (SESPA). Diretoria de Vigilância em Saúde. Departamento de Epidemiologia. Banco de dados de hanseníase do Sistema de Agravos de Notificação. Belém: SESPA, 2017.

27. SECRETARIA DE ESTADO DE SAÚDE PÚBLICA DO PARÁ (SESPA). Hanseníase no Pará: fatores que interferem na manutenção dos elevados coeficientes de detecção. Relatório de Pesquisa Operacional, 2009. Disponível em: https://english.aifo.it/research/leprosy/pesquisa_hanseniase_para.pdf. Acessado em: 8 de abril de 2021.

28. SILVA DML, et al. Tecnologias educacionais na assistência de enfermagem em educação em saúde: revisão integrativa. Rev enferm UFPE on line, 2017; 11(Supl.2): 1044-51. 